\title{
OPEN The acute effect in performing common range of motion tests in healthy young adults: a prospective study
}

\author{
F. Holzgreve ${ }^{1 \bowtie}$, C. Maurer-Grubinger ${ }^{1}$, J. Isaak2 ${ }^{2}$, P. Kokott' ${ }^{2}$, M. Mörl-Kreitschmann ${ }^{2}$, \\ L. Polte ${ }^{2}$, A. Solimann' ${ }^{2}$, L. Wessler' ${ }^{2}$, N. Filmann ${ }^{3}$, A. van Mark ${ }^{1}$, L. Maltry ${ }^{1}$, D. A. Groneberg ${ }^{1}$ \& \\ D. Ohlendorf ${ }^{1}$
}

In the application of range of motion (ROM) tests there is little agreement on the number of repetitions to be measured and the number of preceding warm-up protocols. In stretch training a plateau in ROM gains can be seen after four to five repetitions. With increasing number of repetitions, the gain in ROM is reduced. This study examines the question of whether such an effect occurs in common ROM tests. Twenty-two healthy sport students $(10 \mathrm{~m} / 12 \mathrm{f}$.) with an average age of $25.3 \pm 1.94$ years (average height $174.1 \pm 9.8 \mathrm{~cm}$; weight $66.6 \pm 11.3 \mathrm{~kg}$ and BMI $21.9 \pm 2.0 \mathrm{~kg} / \mathrm{cm}^{2}$ ) volunteered in this study. Each subject performed five ROM tests in a randomized order-measured either via a tape measure or a digital inclinometer: Tape measure was used to evaluate the Fingertipto-Floor test (FtF) and the Lateral Inclination test (LI). Retroflexion of the trunk modified after Janda (RF), Thomas test (TT) and a Shoulder test modified after Janda (ST) were evaluated with a digital inclinometer. In order to show general acute effects within 20 repetitions we performed ANOVA/ Friedman-test with multiple comparisons. A non-linear regression was then performed to identify a plateau formation. Significance level was set at $5 \%$. In seven out of eight ROM tests (five tests in total with three tests measured both left and right sides) significant flexibility gains were observed (FtF: $p<0.001$; LI-left/right: $p<0.001 / 0.001 ; \mathrm{RF}: p=0.009$; ST-left/right: $p<0.001 / p=0.003$; TT-left: $p<0.001$ ). A non-linear regression with random effects was successfully applied on FtF, RF, LI-left/ right, ST-left and TT-left and thus, indicate a gradual decline in the amount of gained ROM. An acute effect was observed in most ROM tests, which is characterized by a gradual decline of ROM gain. For those tests, we can state that the acute effect described in the stretching literature also applies to the performance of typical ROM tests. Since a non-linear behavior was shown, it is the decision of the practitioner to weigh up between measurement accuracy and expenditure. Researchers and practitioners should consider this when applying ROM assessments to healthy young adults.

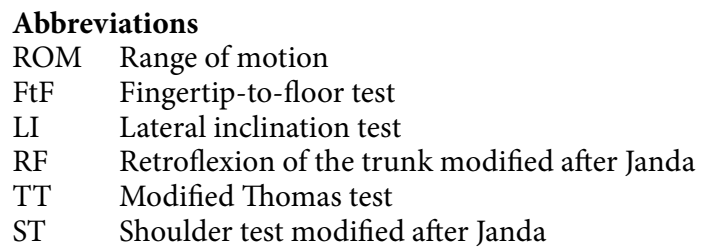

In the application of range of motion (ROM) tests there is little agreement on the number of repetitions to be measured and the number of preceding warm-up protocols. In general, ROM tests are tools to measure joint mobility on the basis of routine (scientific) procedures ${ }^{1-4}$. The aim of any ROM test is to determine the maximum

\footnotetext{
${ }^{1}$ Institute for Occupational Medicine, Social Medicine and Environment Medicine, Goethe-University Frankfurt, Frankfurt Theodor-Stern-Kai 7, Haus 9b, 60590 Frankfurt am Main, Germany. ${ }^{2}$ Institute of Sport Science, Goethe-University Frankfurt, Frankfurt am Main, Germany. ${ }^{3}$ Institute of Biostatistics, Goethe-University Frankfurt, Frankfurt am Main, Germany. ${ }^{\square}$ email: holzgreve@med.uni-frankfurt.de
} 
joint mobility. Hence, in every execution of the test, a stretching stimulus is applied on the connected muscle-tendon units. It has been shown, that in stretch training, such an acute effect occurs after only a few repetitions and manifests itself among other things in increased mobility, stretch tolerance and reduced passive torque ${ }^{4-9}$. It can be expected that this acute effect also occurs during the ROM test. Therefore, differences in the measurement protocol with regard to the measured repetitions and previous warm-up exercises, could lead to different results. However, the current evidence does not allow a determination of precise measurement protocols.

With a reproducible test procedure, the ROM value is a parameter to show changes in flexibility in an intervention. Depending on the joints to be measured, measuring tapes, goniometers or inclinometers are usually applied $^{10-12}$. In general, ROM assessments depict either the active ROM or the passive ROM. To perform active ROM, the person to be tested moves the assessed joint without assistance using the agonistic musculature. In passive ROM, the therapist, the investigator, or another external force, moves the body part of the person to be tested through the ROM ${ }^{1}$. However, changes in passive torque, stretch tolerance and isometric muscle force influence the ROM value.

In static and dynamic stretching, an acute effect occurs within the first five stretches for ROM, stretch tolerance, passive torque and energy, which then return to baseline after $1 \mathrm{~h}^{5,8,9}$. The acute effect of stretching on ROM shows the greatest improvement in the first repetitions, whereas the ROM gain is reduced with increasing repetitions $s^{4,7}$. Consequently, four to five repetitions are recommended for the practical implementation within a stretch training session since subsequent gains are only minimal ${ }^{6,7}$. Accordingly, a logarithmic behaviour can be attributed to the acute effect of stretching on ROM. This is valid for stretching training, but how does it behave when the ROM is to be determined in an assessment setting?

The current literature provides no answers on whether such an effect occurs in the performance of common ROM tests. Scientific practice uses mixed approaches for the problem of acute effects. For example, in interventions and normative data surveys, one to three repetitions were carried out with no to two trials' warm-ups, mostly without any information on rest times ${ }^{13-17}$ or with no information about the number of repetitions ${ }^{18,19}$. In most reliability studies, either one repetition was primarily carried out ${ }^{10,20,21}$ or a warm-up on a bicycle ergometer employed, followed by one to two trials of warm-up prior to the actual measurement being conducted ${ }^{22,23}$. In a reliability study investigating trunk mobility, each subject was instructed to perform five repetitions of each tested motion in advance of the measurement being taken ${ }^{24}$.

In conclusion, there is no homogeneity regarding warm-up, the number of repetitions and averaging in the context of interventions, normative data surveys and reliability studies. Particularly, in light of a possible acute effect of stretching on ROM, a standardized procedure is necessary, since in common stretching the effect is very large, especially in the first five repetitions $s^{4,6,7}$. Due to the inconsistent implementation, in both the practical application and in reliability studies, the presence of an acute effect harms the comparability of ROM test results. Also, if it can be assured, that subjects are in a warmed up state, possible stiffening factors (e.g. sports prior to measurement) can be controlled for.

Thus, the aim of this study was to investigate the repetition-dependent acute effect of stretching on the ROM in the application of ROM tests and whether an equal behaviour can be derived. One further goal was to test whether there is a plateau formation after several repetitions. Therefore, multi-joint movements were chosen because they are restricted by the muscle-tendon unit. Single-joint movement are on the other hand limited by bones (e. g. elbow extension), mass (e. g. knee flexion) or ligaments (e. g. knee extension) ${ }^{25}$. Five frequently used and evaluated multi-joint ROM tests were selected, which mainly evaluate the mobility of the trunk: Fingertipto-Floor test ${ }^{1,26}$, Lateral Inclination test (left and right side) ${ }^{1,27}$, Retroflexion of the trunk after Janda ${ }^{1}$, Shoulder test after Janda (left and right side) $)^{1,28}$ and the modified Thomas test (left and right side) ${ }^{1,10,29}$. On this basis, recommendations can be derived for the practical application of quality criteria.

\section{Material and methods}

Subjects. Twenty-two healthy sports students (10 m/12 f.; $25.3 \pm 1.94$ years; $174.1 \pm 9.8 \mathrm{~cm} ; 66.6 \pm 11.3 \mathrm{~kg}$; $21.9 \pm 2.0 \mathrm{~kg} / \mathrm{m}^{2}$ ) volunteered in this prospective study. Exclusion criteria were relevant operations or surgical stiffening of the musculoskeletal system, a relevant artificial joint replacement, severe diseases such as ankylosing spondylitis, chronic destructive joint diseases, multiple sclerosis, myodystrophic or neurodegenerative diseases, congenital malpositions of the musculoskeletal system or an acute herniated disc. In addition, the intake of muscle relaxants or other drugs that influence the elasticity of the musculature and pregnancy were considered as contra indicators. Two sports students with experience in exercise physiology carried out the measurements; both raters were instructed on the methods and practised until the execution was satisfactory.

All participants provided written informed consent to take part in the study in advance. This study was approved by the ethics research committee of the Goethe-University (2018-46) in Frankfurt am Main, Germany and was conducted in accordance with the 1964 Helsinki Declaration and its later amendments.

Measurement systems and ROM tests. The ROM measurements used in this study, which are described below, were evaluated with either a tape measure (Fingertip-to-Floor and Lateral Inclination test) ${ }^{26,30-33}$ or a digital inclinometer (Retroflexion, Shoulder and Thomas test) ${ }^{10,27,29,34-41}$ (Fig. 1). The digital inclinometer (Model: Acumar Digital Inclinometer Model ACU002 / Lafayette Instrument Company / Lafayette / USA) has a measurement accuracy comparable to a goniometer ${ }^{35}$. As the digital inclinometer shows only integers, the absolute measurement error was set to $0.3^{\circ}$. A detailed description of the measurement tools and the ROM tests can be found in Holzgreve et. al. ${ }^{15}$.

Fingertip-to-floor test (FtF). The FtF test is used to assess the active ROM of the back, both hips, the ischiocrural musculature and the neuromeningeal structures ${ }^{1}$ (Fig. 1a). A tape measure is used to assess the distance 

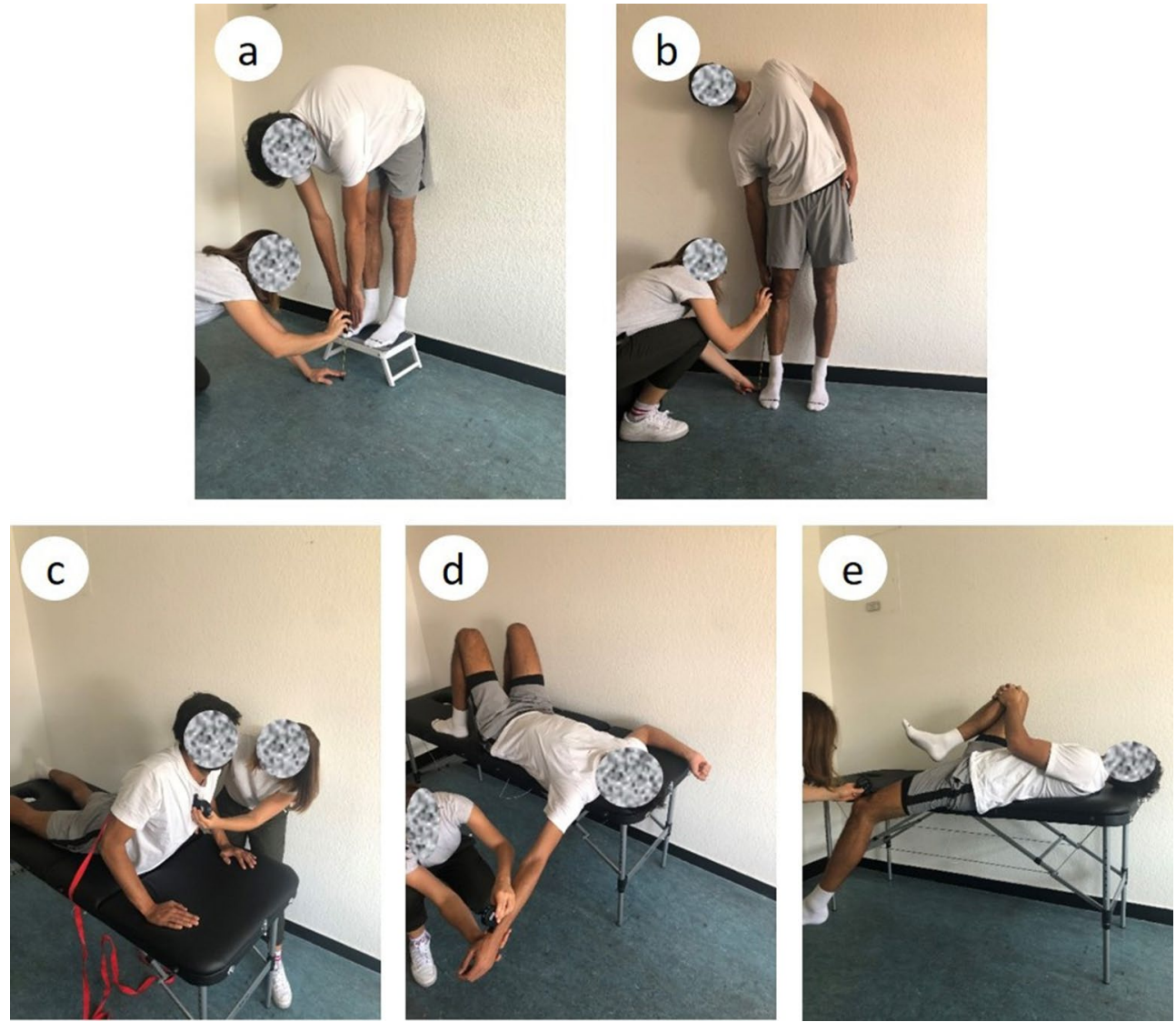

Figure 1. The ROM tests examined in this study. (a) Fingertip-to-Floor test; (b) Lateral Inclination test; (c) Retroflexion of the trunk after Janda in a modified version; (d) Shoulder test modified after Janda; (e) Modified Thomas test.

between the most distal point of the fingers and the floor. Accordingly, a smaller measure corresponds to a greater flexion performance. The reliability of this test lies between $r=0.76$ and $r=0.99^{20,30-32}$ and shows a good sensitivity for changes ${ }^{26}$.

Lateral inclination (LI). This test is performed in a standardized stand position by flexing the upper body in the frontal plane (Fig. 1.b). Sagittal fluctuations in the Lateral Inclination are eliminated by leaning the back against a wall. The trunk lateral flexion active ROM is evaluated with a tape measure measuring the distance between the fingertip and the floor ${ }^{1,27}$ and has an intrarater reliability of $0.95^{42}$.

Retroflexion of the trunk after Janda in a modified version (RF). In order to evaluate the extension of the lumbar and thoracic spine, the modified retroflexion test according to Janda (RF) was performed ${ }^{28}$ (Fig. 1c). The participants lay on a treatment couch with a tensioning strap located at the level of the posterior superior iliac spina, counteracting pelvic rotation in the sagittal plane. With the hands placed next to the shoulders the participants push and extend their elbows as far as possible. The position of the thoracic spine in the sagittal plane is determined by placing the digital inclinometer on the sternum. This test evaluates the spinal active ROM ${ }^{1}$.

Shoulder test modified after Janda (ST). This shoulder mobility test is a modification of the examination after $\operatorname{Janda}^{28}$ (Fig. 1d). The ST is a passive ROM test ${ }^{1}$ which evaluates the mobility of the shoulder joint, especially of the pectoralis major muscle. For this purpose, the subjects where positioned on the bench in a supine position. The arm is extended in $90^{\circ}$ abduction. In contrast to Janda, the elbow is stretched. The digital inclinometer is then placed proximal to the processus styloideus radii on the radius.

Modified Thomas test (TT). The pelvic inclination must be controlled to obtain valid results ${ }^{29}$ (Fig. 1e). In order to standardize the pelvic inclination, the digital inclinometer is placed downwards of the anterior superior iliac spine. In this position, the alignment of the pelvis is set to $0^{\circ}$. The extension inclination is then measured by placing the inclinometer on the thigh above the patella. The modified Thomas test is a widely used passive ROM test to assess the presence of hip flexion contracture and to measure hip extensibility ${ }^{1,29}$. For both the digital inclinometer and goniometer, high interrater reliabilities have been reported $(\mathrm{r}=0.91-0.93 \text {; ICC }=0.89-0.92)^{10}$. The intrarater parallel-forms reliability is also very high with correlations of $r=0.91-0.93$; ICC $=0.89-0.92^{10}$. 


\begin{tabular}{|l|l|l|l|l|l|l|l|l|l|}
\hline & \multirow{2}{*}{$\begin{array}{l}\text { Fingertip-to- floor } \\
\text { test }\end{array}$} & \multicolumn{2}{|l|}{ Lateral Inclination } & \multicolumn{2}{l|}{} & \multicolumn{2}{l|}{ Shoulder test } & \multicolumn{2}{l|}{ Mod. Thomas test } \\
\cline { 7 - 11 } & & Left & Right & Retro-flexion & Left & Right & Left & Right \\
\hline Friedman/ANOVA & $p<0.001^{1}$ & $p<0.001^{1}$ & $p<0.001^{1}$ & $p 0.01^{2}$ & $p<0.001^{1}$ & $p<0.01^{1}$ & $p<0.001^{1}$ & $p>0.05^{1}$ \\
\hline $\begin{array}{l}\text { individuals w/ROM } \\
\text { gain }\end{array}$ & $22 / 22$ & $18 / 22$ & $20 / 22$ & $16 / 22$ & $20 / 22$ & $19 / 22$ & $16 / 22$ & $13 / 22$ \\
\hline $\begin{array}{l}\text { Mean of standard error } \\
\text { of measurement }\end{array}$ & 1.555 & 0.664 & 0.665 & 0.818 & 1.65 & 1.989 & 1.185 & 1.564 \\
\hline $50 \%$ of movement & 5 & 5 & 5 & 2 & 6 & - & 6 & - \\
\hline $75 \%$ of movement & 10 & 9 & 9 & 3 & 11 & - & 11 & - \\
\hline
\end{tabular}

Table 1. P-values of Friedman test and ANOVA for each ROM test. Relative amount of individuals with an ROM gain, mean of the standard measurement error and number of repetitions needed to achieve $50 \%$ and $75 \%$ of the total ROM increase for each ROM test, respectively. ANOVA $=1$; Friedman test $=2$.

Procedure. There was no standardized warm-up, because every repetition represents a specific warm-up. All subjects performed all testing in one day. There was no familiarization prior to the testing. Each subject performed 20 repetitions in each ROM test listed above. The test order was randomized. Each test repetition had to be held on the active or passive maximum ROM (according to the test) for about three seconds. The investigator counted down from three, when the maximum ROM position was reached. At zero, the examiner recorded the ROM according to each test protocol. There was a break of $3 \mathrm{~s}$ between each repetition in each testing session. All measurements were carried out by two raters.

Statistical analysis. Statistical analysis was performed using BiAS version 11.08 (Epsilon-Verlag, Darmstadt, Germany) and R (R Core Team 2019) ${ }^{43}$ and figures were produced using the package ggplot $2^{44}$. Since no such study is known to date from which expected results can be derived and, in particular, no information on the dispersion of the data is available, no formal power analysis is carried out. The study described here is therefore to be regarded as a pilot study. To determine if an acute effect occurs at the individual level, a regression was performed on every individual's performance. Subsequently, the number of subjects with a slope sign corresponding to an ROM gain were counted for each ROM test. According to normal distribution of the data, either univariate ANOVA with repeated measurements ${ }^{45}$ or the Friedman-test ${ }^{46}$ with multiple comparisons were conducted. These tests were performed in order to identify an overall effect and changes within 20 repetitions. Bonferroni correction was used to counteract the problem of multiple comparisons. The mean of the standard error of measurement of each repetition served as a parameter for the measurement accuracy. If the sign test was positive, non-linear regression was applied. Random effects took repeated measurements into account, thus describing the behavior of a potential acute effect of ROM: $a^{*} \exp \left(-b^{*} x\right)+c$.

With "a" being the amplitude at the very beginning, "b" the coefficient describing the change from trial to trial and "c" the asymptotic measure. In case, the model was not adequate (i.e. parameter estimations were nonsignificant), linear regression with random effects was performed to quantify the trend: $a \cdot x+b$.

Significance was set at $5 \%$.

Ethics approval. All participants provided written informed consent to take part in the study in advance. This study was approved by the ethics research committee of the Goethe-University (2018-46) in Frankfurt am Main, Germany.

\section{Results}

Except of TT-right $(p=0.93)$, all ROM tests revealed significant flexibility gains within 20 repetitions, indicating changes of ROM due to repetitive stretching (FtF: $p<0.001$; LI-left/right: $p<0.001 / p<0.001$; RF: $p=0.009$; STleft/right: $p<0.001 / p=0.003$; TT-left: $p<0.001$ ) (Table 1). Furthermore, multiple comparisons revealed the first significant ROM gain in FtF, LI-left/right, RF and ST-left after five to eight repetitions, whereas in ST-right the first significant gain occurred after 19 repetitions. However, TT-left and TT-right showed no significant changes in multiple comparisons. All repetitions were held for $3 \mathrm{~s}$.

In the individual analysis, only FtF showed a uniform effect, indicating a ROM gain for every volunteer. Furthermore, in LI-left/right and ST-left/right more than $80 \%$ of the subjects experienced a ROM gain (Table 1). In contrast, in RF and TT-left/right less than $75 \%$ of the subjects recorded a ROM gain (Table 1).

Furthermore, mean values of the standard error of measurement in contralateral sides differ in ST (1.65/1.989) and TT (1.185/1.564), whereas LI shows almost identical mean values of the standard error of measurement $(0.664 / 0.665)$ (Table 1).

The non-linear regression for FtF, RF, LI-left/right, ST-left and TT-left showed a repetition dependent gradual decline in the ROM gained (Fig. 2). Hence, a plateau formation could be derived from the non-linear regression. As the non-linear model was not adequate for ST-right and TT-right a linear regression with random effects was performed to quantify the trend (Fig. 2). Whereas in ST-right a slope of at least 0.127 of 'a' could be derived, there was no significant difference between 'a' and zero for TT-right. 

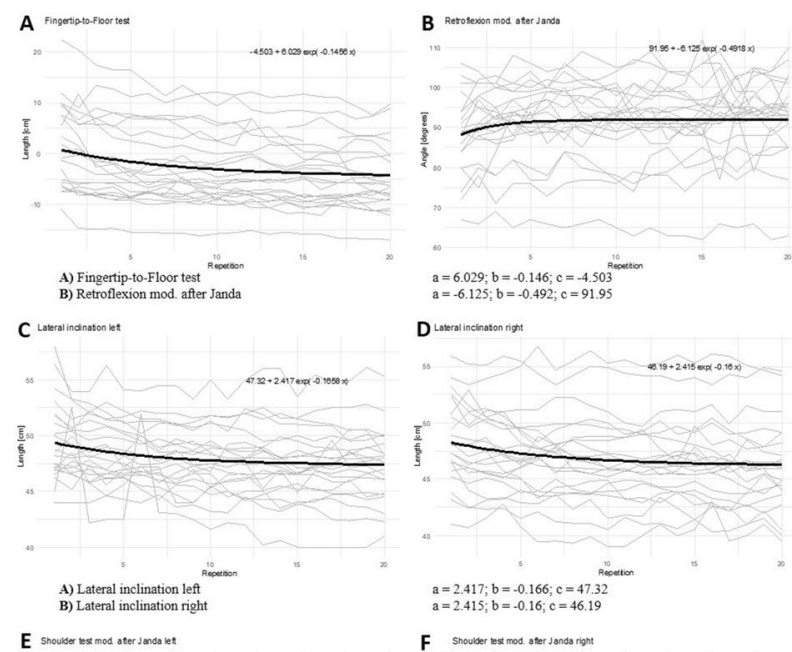

D takatinchaston nigt
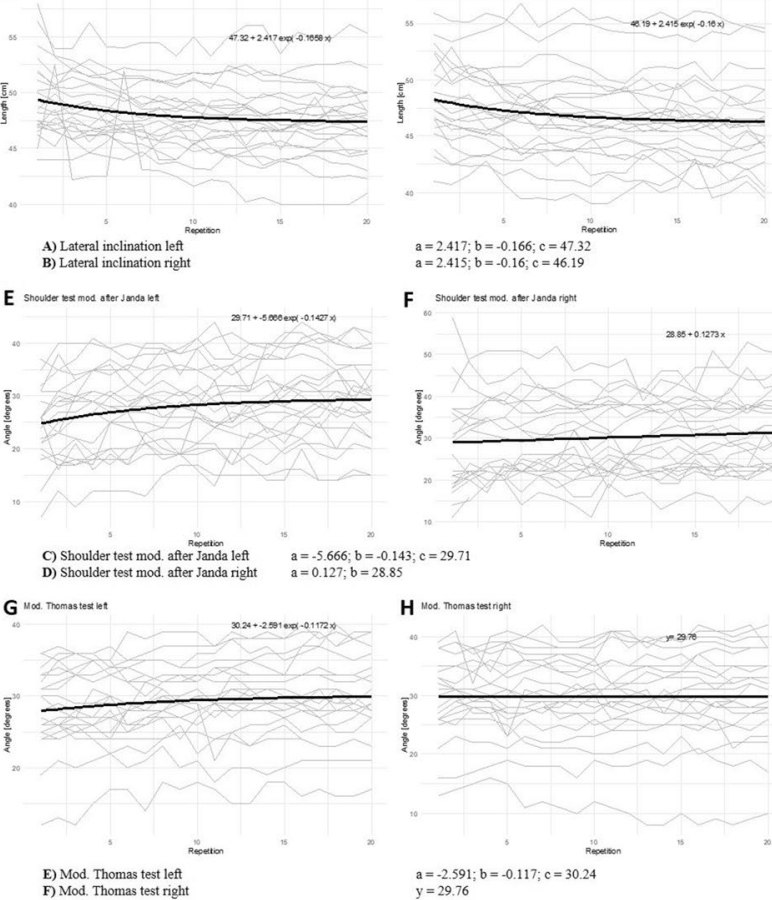

$a=2.417, b=-0.166 ; c=47.32$
$a=2.415 ; b=-0.16 ; c=46.19$

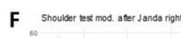
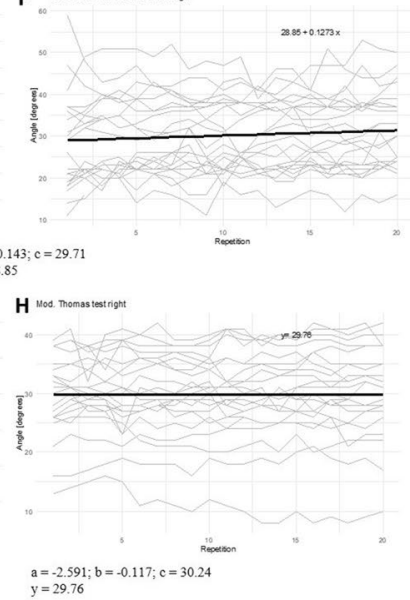

Figure 2. Non-linear regression for FtF, RF, LI, ST left and TT left. Linear regression for ST right and TT right. Parameters of the function are shown below.

\begin{tabular}{|c|c|c|c|c|c|c|c|c|}
\hline \multirow[b]{2}{*}{$\%$ total ROM increase } & \multirow[b]{2}{*}{ Fingertip-to-floor test } & \multicolumn{2}{|c|}{\begin{tabular}{|l|} 
Lateral \\
inclination
\end{tabular}} & \multirow[b]{2}{*}{ Retroflexion } & \multicolumn{2}{|c|}{ Shoulder test } & \multicolumn{2}{|c|}{$\begin{array}{l}\text { Mod. Thomas } \\
\text { test }\end{array}$} \\
\hline & & Left & Right & & Left & Right & Left & Right \\
\hline 25 & 3 & 2 & 2 & 1 & 3 & - & 3 & - \\
\hline 50 & 5 & 5 & 5 & 2 & 6 & - & 6 & - \\
\hline 60 & 7 & 6 & 6 & 2 & 8 & - & 7 & - \\
\hline 70 & 9 & 8 & 8 & 2 & 10 & - & 9 & - \\
\hline 80 & 12 & 10 & 11 & 3 & 13 & - & 12 & - \\
\hline 90 & 17 & 14 & 15 & 4 & 18 & - & 17 & - \\
\hline 95 & 22 & 19 & 19 & 5 & 24 & - & 23 & - \\
\hline 99 & 32 & 28 & 29 & 8 & 36 & - & 34 & - \\
\hline
\end{tabular}

Table 2. Relative ROM gain dependent on the number of repetitions for each ROM test.

The parameter estimates LI-left and -right were significantly different for the parameter 'a' $(p<0.0001)$. Having successfully applied a non-linear regression on six out of the eight ROM tests, it is possible to postulate predictions on the number of repetitions needed to achieve a certain amount of maximal ROM gain (Table 2).

\section{Discussion}

Except of TT-right all ROM tests demonstrated a repetition-dependent change in ROM. In addition, six out of the eight ROM tests (FtF, RF, LI-left/right, ST-left and TT-left) showed a gradual decline in the amount of ROM gained, indicating a plateau formation (Fig. 2). The results confirm the hypothesis that ROM tests provide a stretch stimulus analogous to stretching exercises. They are consistent with repetition-dependent acute effects of stretch training ${ }^{4,6,7,47,48}$ and demonstrate that this effect is also present in typical ROM tests. Based on the 
conducted regression, a plateau could be derived for each ROM test, which depicts an area where ROM gain is very small and negligible with each further repetition ${ }^{4}$. The increase in mobility lays between 1 and 6 degrees and 2 and $6 \mathrm{~cm}$, respectively (Fig. 2). Thus, it can be stated that a different number of repetitions performed in mobility assessment settings lead to different angle values for the ROM (Fig. 2).

For the practical application of these six ROM tests, the regression provided information on the number of repetitions above which the test offers reliable values for the evaluated ROM (Table 2). This is especially the case when different or unknown warm-up states are employed; the execution of several repetitions according to the regression can allow for a better standardization. The number of repetitions required for a certain increase in ROM is very similar amongst ROM tests which supports the stability of the applied methods.

With respect to the practical application, we recommend to assess in the area of the plateau of each test. Yet a concrete naming of a plateau should be avoided since, even in higher stretch repetitions, small gains were recognizable. However, increasing the ROM from $50 \%$ of maximal to $60 \%$ of maximal required only one or two repetitions (e. g. FtF/LI in Table 2). When approaching the plateau, more repetitions were needed to gain the same amount of ROM, for instance, to increase from 80 to $90 \%$ up to five additional repetitions were necessary (Table 2). The respective plateau formation can be derived descriptively from Fig. 2. Only the RF differed significantly from the other results. The goodness of fit of RF (adjusted R squared: 0.606; root mean square error: 0.746 ) was clearly worse than those in the other tests; this may be due to the fact that performing the RF requires maximum elbow extension, therefore, fatigue effects cannot be excluded when performing 20 maximum repetitions.

Although the type of stretching used in all ROM tests was static, the duration differed greatly from the duration used in static stretching. While in common ROM tests the subjects keep their positions for seconds, recommendations for static stretching range from five seconds to $15 \mathrm{~min}$ or even more ${ }^{49-54}$. Boyce and Brosky ${ }^{6}$ showed a plateau occurrence for $15 \mathrm{~s}$ static stretching at the fifth through to the tenth stretch repetition.

The reason why an overall effect on the individual level could not be shown is probably because the effects are very small in comparison to the measurement accuracy. In total, the range of variation of the measurements is large, especially for the digital inclinometer which only uses integers. Here, one sees differences favoring the tape measure over the digital inclinometer regarding individual performances.

In the present investigation, only the regression curve of RF (Fig. 2) appeared to match the proposed plateau formation timing. In the FtF, the plateau formation ( $80 \%$ of the total ROM increase) of the LI-left and right, STleft and TT-left occurred between the 10th and 13th stretch repetition (Table 2). The largely uniformly delayed plateau formation compared to Boyce and Brosky's $s^{6}$ may be explained by a significantly shorter stimulus exposure time. There appears to be dose-dependent differences, although the underlying effect is the same. The fact that in RF the plateau was already reached by the fifth stretch repetition, may be a result of fatigue effects occurring in performing this ROM test. Furthermore, RF has less muscle inhibition of the movement compared to the other ROM tests investigated. The spine mobility is mainly limited by ligaments, especially the ligamentum longitudinale anterius concerning hyperextension ${ }^{55}$. Due to their collagen structure, ligaments have only limited stiffness $^{56}$. A limitation of motion due to ligament inhibition may explain premature plateau formation in RF.

Tests such as FtF, TT and LI displayed high intrarater reliabilities (FtF: $r=0.76-0.99^{26,29-32}$; TT: $r=0.89-0.92^{10,29}$; LI: $r=0.95^{42}$, which were mostly determined in the first trial over two days ${ }^{10,20,21}$. Due to mostly good reliabilities, it can be assumed that the warm-up state of the subjects was standardized. Nevertheless, it is unclear as to how sensitive the measurement of the first repetition is for changes, e.g. in the context of an intervention. Does the first repetition represent the actual mobility? In addition, the question arises as to what extent sample measurements, different warm-up states, special warm-ups or faulty measurements with subsequent second repetitions falsify the results. Since the influence of additional repetitions on the ROM in the context of ROM tests has scarcely been investigated so far, reference must be made at this point to further, future investigations. Angle values obtained from protocols that differ in the application of the number of repetitions, preliminary warm-up routines or averaging must not be compared. It should be noted that the functions (Fig. 2) provide the basis for a mobility measurement on the plateau. This could serve as a basis for the comparability of ROM tests.

No exponential regression could be performed in ST-right and TT-right, whereas it could be applied for the contralateral side. Considering the mean values of the standard error of measurement for each ROM test, Table 1 shows discrepancies among contralateral sides in ST and TT. The mean standard error of measurement of the right side was considerably greater; this may explain the inconsistent data for the right side in ST and TT. In both tests, which measure the mobility of extremities, the right side showed little or no difference. The discrepancy may be due to the one-sided distribution of handedness and footedness. Biomechanical differences have been shown between left- and right-handed baseball pitchers and also for the elbow flexion, horizontal glenohumeral abduction and wrist coronal plane motion ${ }^{57}$. Furthermore, discrepancies in the test protocol existed in TT and ST compared to FtF, LI and RF. In TT there was no offset visible between the measurements during the test execution. The subjects remained in a permanent strain with sub-maximal intensity. The duration of stimulus exposure was, therefore, significantly longer than in the active ROM tests, in which the stimulus was maintained for about $3 \mathrm{~s}$. In addition, in ST the arm to be measured was moved through and repositioned after each measurement repetition. However, these discrepancies do not explain different contralateral results, but may lead to different intertest outcomes There are further differences between the ROM tests in the different strain methods implicitly used in the test execution that may lead to different outcomes. If the ROM tests are categorized according to the properties and effects of stretching, then differences between the tests become apparent (Table 3).

Differences in either active or passive stretching are particularly remarkable ${ }^{1}$. While FtF, LI and RF tested at maximum torque, ST and TT tested at sub-maximal torque. The source of torque in ST and TT derived solely from gravity and depended on the weight, length and weight distribution of the lever-arm. As the effects between maximal and sub-maximal stretching on the ROM differed ${ }^{47}$, this, may explain the discrepancies.

As our subjects were young healthy adults, further studies are needed to exploit whether this effect does also occur in impaired populations or in the elderly. 


\begin{tabular}{|c|c|c|c|c|c|}
\hline & Fingertip-to-floor & Lateral inclination & Retroflexion & Shoulder test & Thomas test \\
\hline Stretching type & Static & Static & Static & Static & Static \\
\hline Torque type & Active & Active & Active & Passive & Passive \\
\hline Source of torque & Antagonist and gravity & Antagonist and gravity & $\begin{array}{l}\text { Antagonist and elbow } \\
\text { extension }\end{array}$ & Gravity & Gravity \\
\hline Intensity & Maximal & Maximal & Maximal & Sub-maximal & Sub-maximal \\
\hline $\begin{array}{l}\text { Physiological cause for } \\
\text { increased ROM }\end{array}$ & $\begin{array}{l}\text { Muscle/tendon stiffness } \downarrow \\
\text { stretch tolerance } \uparrow\end{array}$ & $\begin{array}{l}\text { Muscle/tendon stiffness } \downarrow \\
\text { stretch tolerance } \uparrow\end{array}$ & $\begin{array}{l}\text { Muscle/tendon stiffness } \downarrow \\
\text { stretch tolerance } \uparrow\end{array}$ & Muscle/tendon stiffness $\downarrow$ & Muscle/tendon stiffness $\downarrow$ \\
\hline
\end{tabular}

Table 3. Stretching properties (stretching type, torque type, source of torque, intensity and physiological cause for increased ROM) for each ROM test.

\section{Conclusion}

An acute effect was observed in most ROM tests, which is characterized by a gradual decline in the amount of ROM gain. Thus, the same number of repetitions is required for the increase in ROM of $0-50 \%$ as for the increase of $50-75 \%$ of the total ROM increase. The behaviour of this acute effect could be determined using a non-linear regression for most of the ROM tests. For these tests, we can state that the acute effect described in the stretching literature of ROM also applies to the performance of typical ROM tests. Researchers and practitioners should consider this when applying ROM assessments to young healthy adults.

\section{Data availability}

There are no further data or materials than shown in this manuscript.

Received: 16 January 2020; Accepted: 17 November 2020

Published online: 10 December 2020

\section{References}

1. Clarkson, H. M. Joint Motion and Function Assessment: A Research-Based Practical Guide (Lippincott Williams \& Wilkins, Philadelphia, 2005).

2. Shelbourne, K. D. \& Klotz, C. What I have learned about the ACL: utilizing a progressive rehabilitation scheme to achieve total knee symmetry after anterior cruciate ligament reconstruction. J. Orthopaedic Sci. 11, 318 (2006).

3. Verrall, G. M. et al. Hip joint range of motion restriction precedes athletic chronic groin injury. J. Sci. Med. Sport 10, 463-466 (2007).

4. Glück, S., Hoffmann, U., Schwarz, M. \& Wydra, G. Range of motion, traction force and muscle activity in self- and externalregulated stretching. Deutsche ZeitschriftfürSportmedizin 53, 66-71 (2002).

5. Magnusson, S. P., Simonsen, E. B., Aagaard, P. \& Kjaer, M. Biomechanical responses to repeated stretches in human hamstring muscle in vivo. Am. J. Sports Med. 24, 622-628. https://doi.org/10.1177/036354659602400510 (1996).

6. Boyce, D. \& Brosky, J. A. Determining the minimal number of cyclic passive stretch repetitions recommended for an acute increase in an indirect measure of hamstring length. Physiother. Theory Pract. 24, 113-120. https://doi.org/10.1080/09593980701378298 (2008).

7. Taylor, D. C., Dalton, J. D., Seaber, A. V. \& Garrett, W. E. Viscoelastic properties of muscle-tendon units. The biomechanical effects of stretching. Am. J. Sports Med. 18, 300-309. https://doi.org/10.1177/036354659001800314 (1990).

8. Nakamura, M., Ikezoe, T., Takeno, Y. \& Ichihashi, N. Time course of changes in passive properties of the gastrocnemius muscletendon unit during 5 min of static stretching. Manual Therapy 18, 211-215 (2013).

9. Ryan, E. D. et al. Determining the minimum number of passive stretches necessary to alter musculotendinous stiffness. J. Sports Sci. 27, 957-961 (2009).

10. Clapis, P. A., Davis, S. M. \& Davis, R. O. Reliability of inclinometer and goniometric measurements of hip extension flexibility using the modified Thomas test. Physiother. Theory Pract. 24, 135-141. https://doi.org/10.1080/09593980701378256 (2008).

11. Ekedahl, H., Jönsson, B. \& Frobell, R. B. Fingertip-to-floor test and straight leg raising test: validity, responsiveness, and predictive value in patients with acute/subacute low back pain. Arch. Phys. Med. Rehabil. 93, 2210-2215. https://doi.org/10.1016/j. apmr.2012.04.020 (2012).

12. Oesch, P. et al. Assessments in der Rehabilitation. Band 2: Bewegungsapparat (2. vollständig überarbeitete und erweiterte Auflage). (Hogrefe AG, 2011).

13. Faes, Y. et al. Acute effects of partial-body vibration in sitting position. World J. Orthop. 9, 156-164. https://doi.org/10.5312/wjo. v9.i9.156 (2018).

14. Harvey, D. Assessment of the flexibility of elite athletes using the modified Thomas test. Br. J. Sports Med. 32, 68-70 (1998).

15. Holzgreve, F. et al. The office work and stretch training (OST) study: an individualized and standardized approach for reducing musculoskeletal disorders in office workers. J. Occup. Med. Toxicol. 13, 37. https://doi.org/10.1186/s12995-018-0220-y (2018).

16. Mosler, A. B. et al. Hip strength and range of motion: Normal values from a professional football league. J. Sci. Med. Sport 20, 339-343. https://doi.org/10.1016/j.jsams.2016.05.010 (2017).

17. Segal, N. A., Hein, J. \& Basford, J. R. The effects of Pilates training on flexibility and body composition: an observational study. Arch. Phys. Med. Rehabil. 85, 1977-1981 (2004).

18. Lemos, T. V., Albino, A. C., Matheus, J. P. \& Barbosa Ade, M. The effect of kinesio taping in forward bending of the lumbar spine. J. Phys. Therapy Sci. 26, 1371-1375. https://doi.org/10.1589/jpts.26.1371 (2014).

19. Mengshoel, A. M. \& Robinson, H. S. Clinical significance of specific spinal mobilization for patients with ankylosing spondylitis evaluated by quantitative assessments and patient interviews. Disabil.Rehabil. 30, 355-364. https://doi.org/10.1080/0963828070 1270711 (2008).

20. Perret, C. et al. Validity, reliability, and responsiveness of the fingertip-to-floor test. Arch. Phys. Med. Rehabil. 82, 1566-1570. https ://doi.org/10.1053/apmr.2001.26064 (2001).

21. Wakefield, C. B., Halls, A., Difilippo, N. \& Cottrell, G. T. Reliability of goniometric and trigonometric techniques for measuring hip-extension range of motion using the modified Thomas test. J. Athlet. Train. 50, 460-466. https://doi.org/10.4085/1062-605050.2.05 (2015). 
22. Nussbaumer, S. et al. Validity and test-retest reliability of manual goniometers for measuring passive hip range of motion in femoroacetabular impingement patients. BMC Musculoskelet. Disorders 11, 194. https://doi.org/10.1186/1471-2474-11-194 (2010).

23. Robinson, H. S. \& Mengshoel, A. M. Assessments of lumbar flexion range of motion: intertester reliability and concurrent validity of 2 commonly used clinical tests. Spine 39, E270-275. https://doi.org/10.1097/brs.0000000000000131 (2014).

24. Frost, M., Stuckey, S., Smalley, L. A. \& Dorman, G. Reliability of measuring trunk motions in centimeters. Phys. Ther. 62, 1431-1437. https://doi.org/10.1093/ptj/62.10.1431 (1982).

25. Wiemann, K. Stretching. Grundlagen, Möglichkeiten. Grenzen. Sportunterricht 42, 91-106 (1993).

26. Heikkila, S., Viitanen, J. V., Kautiainen, H. \& Kauppi, M. Sensitivity to change of mobility tests; effect of short term intensive physiotherapy and exercise on spinal, hip, and shoulder measurements in spondyloarthropathy. J. Rheumatol. 27, 1251-1256 (2000).

27. Petherick, M., Rheault, W., Kimble, S., Lechner, C. \& Senear, V. Concurrent validity and intertester reliability of universal and fluid-based goniometers for active elbow range of motion. Phys. Ther. 68, 966-969 (1988).

28. Page, P., Frank, C. C. \& Lardner, R. Assessment and Treatment of Muscle Imbalance: The Janda Approach (Human Kinetics, Katowice, 2010).

29. Vigotsky, A. D. et al. The modified Thomas test is not a valid measure of hip extension unless pelvic tilt is controlled. PeerJ 4, e2325. https://doi.org/10.7717/peerj.2325 (2016).

30. Gauvin, M. G., Riddle, D. L. \& Rothstein, J. M. Reliability of clinical measurements of forward bending using the modified fingertipto-floor method. Phys. Ther. 70, 443-447 (1990).

31. Gill, K., Krag, M. H., Johnson, G. B., Haugh, L. D. \& Pope, M. H. Repeatability of four clinical methods for assessment of lumbar spinal motion. Spine 13, 50-53 (1988).

32 Kippers, V. \& Parker, A. W. Toe-touch test. A measure of its validity. Phys. Therapy 67, 1680-1684 (1987).

33. Merritt, J. L., McLean, T. J., Erickson, R. P. \& Offord, K. P. Measurement of trunk flexibility in normal subjects: reproducibility of three clinical methods. Mayo Clin. Proc. 61, 192-197 (1986).

34. Antonaci, F., Ghirmai, S., Bono, G. \& Nappi, G. Current methods for cervical spine movement evaluation: a review. Clin. Exp. Rheumatol. 18, S45-52 (2000).

35. Bierma-Zeinstra, S. M. et al. Comparison between two devices for measuring hip joint motions. Clin. Rehabil. 12, 497-505. https ://doi.org/10.1191/026921598677459668 (1998).

36. Green, S., Buchbinder, R., Forbes, A. \& Bellamy, N. A standardized protocol for measurement of range of movement of the shoulder using the Plurimeter-V inclinometer and assessment of its intrarater and interrater reliability. Arthritis Care Res. Off. J. Arthritis Health Profess. Assoc. 11, 43-52 (1998).

37. Hole, D. E., Cook, J. M. \& Bolton, J. E. Reliability and concurrent validity of two instruments for measuring cervical range of motion: effects of age and gender. Manual Therapy 1, 36-42. https://doi.org/10.1054/math.1995.0248 (1995).

38. Hoving, J. et al. How reliably do rheumatologists measure shoulder movement?. Ann. Rheum. Dis. 61, 612-616. https://doi. org/10.1136/ard.61.7.612 (2002).

39. Kolber, M. J., Saltzman, S. B., Beekhuizen, K. S. \& Cheng, M. S. Reliability and minimal detectable change of inclinometric shoulder mobility measurements. Physiother. Theory Pract. 25, 572-581. https://doi.org/10.3109/09593980802667995 (2009).

40. Rheault, W., Miller, M., Nothnagel, P., Straessle, J. \& Urban, D. Intertester reliability and concurrent validity of fluid-based and universal goniometers for active knee flexion. Phys. Ther. 68, 1676-1678 (1988).

41. Valentine, R. E. \& Lewis, J. S. Intraobserver reliability of 4 physiologic movements of the shoulder in subjects with and without symptoms. Arch. Phys. Med. Rehabil. 87, 1242-1249. https://doi.org/10.1016/j.apmr.2006.05.008 (2006).

42. Jonsson, E., Ljungkvist, I. \& Hamberg, J. Standardized measurement of lateral spinal flexion and its use in evaluation of the effect of treatment of chronic low back pain. Upsala J. Med. Sci. 95, 75-86. https://doi.org/10.3109/03009739009178578 (1990).

43. R Core Team. R: A language and environment for statistical computing (R Core Team, Vienna 2019).

44. Wickham, H. ggplot2: Elegant Graphics for Data Analysis (Springer, Berlin, 2016).

45. Bortz, J. Statistik: für Human- und Sozialwissenschaftler 411-436 (Springer, Berlin, 2005).

46. Friedman, M. The use of ranks to avoid the assumption of normality implicit in the analysis of variance. J. Am. Stat. Assoc. 32, 675-701. https://doi.org/10.1080/01621459.1937.10503522 (1937).

47. Marshall, F. Effects of different stretch-intensity on the acute change of range of motion. Ger. J. Sports Med. 1, 5-9 (1999).

48. Wydra, G., Glück, S. \& Roemer, K. Short-term effects of singular muscle-stretching-procedures. Ger. J. Sports Med. 1, 10-16 (1999).

49. Wydra, G., Bös, K. \& Karisch, G. The effectiveness of different stretching techniques. Ger. J. Sports Med. 42, 386-400 (1991).

50. Anderson, B. Stretching: 20th Anniversary (Shelter Publications, Bolinas, 2000).

51. Hartley-O’Brien, S. J. Six mobilization exercises for active range of hip flexion. Res. Q. Exerc. Sport 51, 625-635. https://doi. org/10.1080/02701367.1980.10609323 (1980).

52. Sölveborn, S. A. Das Buch vom Stretching: Beweglichkeitstraining durch Dehnen und Strecken. (Mosaik-Verlag, 1989).

53. Bohannon, R. W., Chavis, D., Larkin, P., Lieber, C. \& Riddick, L. Effectiveness of repeated prolonged loading for increasing flexion in knees demonstrating postoperative stiffness. A clinical report. Phys. Therapy 65, 494-496. https://doi.org/10.1093/ptj/65.4.494 (1985).

54. Bohannon, R. W. Effect of repeated eight-minute muscle loading on the angle of straight-leg raising. Phys. Ther. 64, 491-497. https ://doi.org/10.1093/ptj/64.4.491 (1984).

55. Bozkurt, M. in Musculoskeletal Research and Basic Science Vol. 1 (ed Feza Korkusuz) Ch. 7, 761 (Springer, Berlin, 2016).

56. Chino, K. \& Takahashi, H. The association of muscle and tendon elasticity with passive joint stiffness: in vivo measurements using ultrasound shear wave elastography. Clin. Biomech. (Bristol, Avon) 30, 1230-1235. https://doi.org/10.1016/j.clinbiomec h.2015.07.014 (2015)

57. Solomito, M. J., Ferreira, J. V. \& Nissen, C. W. Biomechanical differences between left- and right-handed baseball pitchers. Sports Biomech. 16, 143-151. https://doi.org/10.1080/14763141.2016.1186725 (2017).

\section{Acknowledgements}

All authors confirm that all methods were carried out in accordance with relevant guidelines and regulations.

\section{Author contributions}

F.H. and C.M. developed the theory and performed the computations. F.H.; L.M. and D.O. devised the project, the main conceptual ideas and proof outline. C.M. worked out almost all of the technical details. N.F. verified the analytical Methods. F.H.; L.W.; L.P.; P.K.; A.S.; J.I.; M.M.K.; L.M. and D.O. carried out the experiment. F.H. wrote the manuscript with support from C.M.; N.F.; L.M.; A.v.M. and D.A.G. D.A.G. and D.O. helped supervise the Project. C.M. and N.F. developed the theoretical formalism, performed the analytic calculations and performed the numerical simulations. All authors discussed the results and contributed to the final manuscript.

\section{Funding}

Open Access funding enabled and organized by Projekt DEAL. 


\section{Competing interests}

The authors declare no competing interests.

\section{Additional information}

Correspondence and requests for materials should be addressed to F.H.

Reprints and permissions information is available at www.nature.com/reprints.

Publisher's note Springer Nature remains neutral with regard to jurisdictional claims in published maps and institutional affiliations.

(c) (i) Open Access This article is licensed under a Creative Commons Attribution 4.0 International License, which permits use, sharing, adaptation, distribution and reproduction in any medium or format, as long as you give appropriate credit to the original author(s) and the source, provide a link to the Creative Commons licence, and indicate if changes were made. The images or other third party material in this article are included in the article's Creative Commons licence, unless indicated otherwise in a credit line to the material. If material is not included in the article's Creative Commons licence and your intended use is not permitted by statutory regulation or exceeds the permitted use, you will need to obtain permission directly from the copyright holder. To view a copy of this licence, visit http://creativecommons.org/licenses/by/4.0/.

(C) The Author(s) 2020 